\title{
THE COMMUTATIVITY OF RINGS WITH LEFT MULTIPLICATIVE
}

\section{GENERALIZED DERIVATIONS}

\section{K. CHENNAKESAVULU ${ }^{1}$, C. JAYA SUBBA REDDY ${ }^{2}$,}

\section{A. SIVA KAMESWARA KUMAR ${ }^{3} \&$ K. NAGESH ${ }^{4}$}

${ }^{I}$ Department of Mathematics, S. K. College of Agricultural Sciences, Anantapuramu, Andhra Pradesh, India

${ }^{2}$ Asssistant Professor, Department of Mathematics, S. V. University, Tirupati, Andhra Pradesh, India

${ }^{3}$ Department of Mathematics, B. I. T institute of technology, Hindupur, Anantapuramu, Andhra Pradesh, India

${ }^{4}$ Research Scholar, Department of Mathematics, Rayalaseema University, Karnool, Andhra Pradesh, India

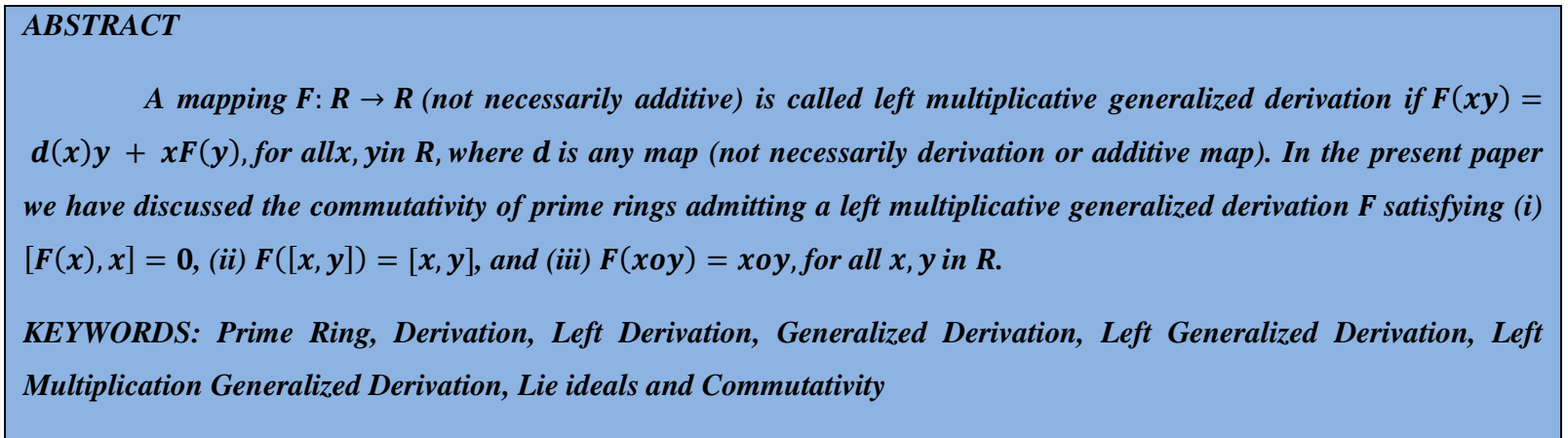

Received: Sep 24, 2018; Accepted: Oct 14, 2018; Published: Nov 03, 2018; Paper Id.: IJESRDEC20188

\section{INTRODUCTION}

\section{Preliminaries}

Throughout this paper $R$ will be denotes an associative ring with the center $Z(R)$. If $n>1$, a ring $R$ is said to be $n$ - torsion free, if for $x \in R, n x=0$ implies $x=0$. Recall that a ring $R$ is called prime if for any $x, y \in R, x R y=\{0\}$ implies that either $x=0$ or $y=0$. An additive mapping $d: R \rightarrow R$ is called a derivation if $d(x y)=d(x) y+x d(y)$, for all $x, y \in R$. An additive mapping $d: R \rightarrow R$ is called a left derivation if $(x y)=$ $x d(y)+y d(x)$, for all $x, y \in R$. An additive mapping $F: R \rightarrow R$ is called a generalized derivation if there exists a derivation $d: R \rightarrow R$ such that $F(x y)=F(x) y+x d(y)$, for all $x, y \in R$. An additive mapping $F: R \rightarrow R$ is called a left generalized derivation if there exists a derivation $d: R \rightarrow R$ such that $F(x y)=x F(y)+d(x) y$, for all for all $x, y \in R$. A mapping $F: R \rightarrow R$ (not necessarily additive) is called a left multiplication generalized derivation $F(x y)=x F(y)+d(x) y$, for all for all $x, y \in R$ where $d$ is any map (not necessarily additive map or derivation). An additive subgroup $U$ of $R$ is to a lie ideal of $R$ if $[U, R] \subseteq U$. Where $[x, y]$ denotes the lie product $x y-y x$ of $x$ and $y$. A mapping $f: R \rightarrow R$ is called centralizing on $S$ if $[f(x), x] \in Z$ for all $x \in S$ and is called commuting on $S$ if $[F(x), x]=0$ for all $x \in S$. Throughout the present paper we shall make extensive use of the following basic commutator identities without any specific mention:

$$
\begin{aligned}
& {[x y, z]=x[y, z]+[x, z] y ;[x, y z]=y[x, z]+[x, y] z} \\
& x o(y z)=(x o y) z-y[x, z]=y(x o z)+[x, y] z
\end{aligned}
$$


$(x y) o z=x(y o z)-[x, z] y=(x o z) y+x[y, z]$.

The study of the commutativity of prime rings with derivations was initiated by E. C. Posner [13]. Hvala [10] introduced the notions of generalized derivations in rings. Mayne J. H [11] studied the centralizing mappings of prime rings. Daif. M. N and Bell. H. E [7] established that if in a semiprime ring $R$ there exists a nonzero ideal $I$ of $R$ and a derivation $d$ such that $d([x, y])=[x, y]$ for all $x, y \in I$, then $I \subseteq Z(R)$. Bresar. M [5] studied commuting traces of bi additive mappings, commutativity- preserving mappings and lie mappings. Awtar. R [2] lie structure in prime rings with derivations. Ashraf. M and Rehman. N [1] extended the mentioned result for lie ideals of $R$. Subba Reddy et al. [14-17] studied left multiplicative generalized derivations in prime and semi prime rings. Nadeem-ur-Rehman [12] studied on commutativity of rings with generalized derivations. In this paper we extended some results on commutativity of rings with left multiplicative generalized derivations.

- Lemma 1: If $U \nsubseteq Z(R)$ is lie ideal of a 2-torsion free prime ring $R$ and $a, b \in R$ such that $a \cup b=0$, then $a=0$ or $b=0$.

Proof: An ideal $M$ of $R$ such that $[M, R] \not \subset Z$ but $[M, R] \subset U$. If $u \in U, m \in M$, and $y \in R$ then $[m a u, y] \in$ $[M, R] \subset U$, thus $0=a[m a u, y] b=a[m a, y] u b+a m a[u, y] b=a(\operatorname{may}-y m a) u b=a m a y u b \quad$, since $a[u, y] b \in a \cup b=0$. Thus $a M a R \cup b=0$. If $a \neq 0$, since $R$ is prime we get $U b=0$; so, if $x \in R, u \in U$ then $(u x-x u) \in U$, whence $(u x-x u) b=0$, and so $u x b=0$. In other words, $u R b=0$; since $U \neq 0$, we get $b=0$.

- Lemma 2: Let $R$ be a 2-torsion free prime ring and $U$ a nonzero lie ideal of $R$. If $d$ is a nonzero derivation of $R$ such that $d(U)=0$, then $U \subseteq Z(R)$.

Proof: Let $u \in U, x \in R$; since $d(u)=0$ and $d(u x-x u)=0$, we get

$u d(x)-d(x) u=d(u x-x u)=0$.

Therefore $u$ is a centralizer $d(R)$. We have $u \in Z$; hence $U \subset Z$.

- Lemma 3 ([2, Theorem 7]): Let $R$ be a 2-torsion free prime ring and $U$ a nonzero lie ideal of $R$. If $d$ is a nonzero derivation of $R$ such that $[u, d(u)] \in Z(R)$ for all $\in U$, then $U \subseteq Z(R)$.

- Lemma 4 ([3, Theorem 4]): Let $R$ be a prime ring and $I$ a nonzero left ideal of $R$. If $R$ admits a nonzero derivation $d$ such that $[x, d(x)]$ is central for all $x \in I$, then $R$ is commutative.

- Lemma 5: If $R$ is a prime ring contains a nonzero commutative right ideal $I$, then $R$ is a commutative.

Proof: If $x \in I$, then $I_{x}(I)=[x, I]=0$. Since $I$ is commutative then $I_{x}=0$ on $R$ and $x$ is a centre. Thus $[x, R]=$ 0 , for every $x \in I$. Hence $I_{a}(I)=0$, for all $a \in R$. Since $I$ is commutative then again $I_{a}=0$ and $a$ is a centre, for all $a \in R$. Therefore $R$ is a commutative.

- Lemma 6: Let $R$ be a 2-torsion free prime ring and $U$ a nonzero lie ideal of $R$. If $U$ is a commutative lie ideal of $R$, i.e. $[u, v]=0$, for all $u, v \in U$, then $U \subseteq Z(R)$.

Proof: Since $U$ is a commutative lie ideal of $R$, i.e. $[u, v]=0$, for all $u, v \in U$.

Replacing $v$ by $[u, r]$ in above equation then, we get 
$[u,[u, r]]=0$, for all $u \in U, r \in R$.

Again replace $r$ by $r s$ in above equation then, we get

$[u,[u, r s]]=0$, for all $u \in U, r, s \in R$.

$[u,[u, r]] s+r[u,[u, s]]+2[u, r][u, s]=0$, for all $u \in U$ and $r, s \in R$.

$2[u, r][u, s]=0$, for all $u \in U$ and $r, s \in R$.

Since $\operatorname{char}(R) \neq 2$, we get

$[u, r][u, s]=0$, for all $u \in U$ and $r, s \in R$.

Replacing $s$ by $s r$ in above equation then, we get

$[u, r][u, s r]=0$

$[u, r] s[u, r]+[u, r][u, s] r=0$

$[u, r] R[u, r]=0$, for all $u \in U$ and $r \in R$.

Thus primness of $R$ forces that $[u, r]=0$, for all $u \in U, r \in R$, and hence $U \subseteq Z(R)$.

\section{Theorem 1}

Let $R$ be a 2-torsion free prime ring and $U$ a nonzero lie ideal of $R$ such that $u^{2} \in U$, for all $u \in U$. If $R$ admits a nonzero left multiplicative generalized derivation $F$ with $d$ such that $[F(u), u]=0$, for all $u \in U$, and if $d \neq 0$, then $U \subseteq Z(R)$.

\section{Proof}

We have, $[F(u), u]=0$, for all $u \in U$

Linearizing equation (1) and using equation (1), we get

$[F(u+v), u+v]=0$

$[F(u)+F(v), u+v]=0$

$[F(u), u]+[F(u), v]+[F(v), u]+[F(v), v]=0$

$[F(u), v]+[F(v), u]=0$, for all $u, v \in U$.

Notice that $v w+w v=(v+w)^{2}-v^{2}-w^{2}$, for all $v, w \in U$.

Since $u^{2} \in U$, for all $u \in U, v w+w v \in U$. Also $v w-w v \in U$, for all $v, w \in U$.

Hence we find that $2 v w \in U$, for all $v, w \in U$.

Replacing $v$ by $2 u v$ in equation (2) and use equations (1) and (2) then, we get

$[F(u), 2 u v]+[F(2 u v), u]=0$, for all $u, v \in U$.

$[F(u), 2 u v]+[2 u F(v)+2 d(u) v, u]=0$ 
$2 u[F(u), v]+2[F(u), u] v+2[u F(v), u]+2[d(u) v, u]=0$

$2 u[F(u), v]+2[F(u), u] v+2 u[F(v), u]+2[u, u] F(v)+2 d(u)[v, u]+2[d(u), u] v=0$

$2[d(u), u] v+2 d(u)[v, u]=0$

Since $R$ is a 2 -torsion free then, we get

$[d(u), u] v+d(u)[v, u]=0$, for all $u, v \in U$

Replacing $v$ by $2 v w$ in equation (3) and using equation (3) then, we get

$[d(u), u] 2 v w+d(u)[2 v w, u]=0$

$[d(u), u] 2 v w+2 d(u) v[w, u]+2 d(u)[v, u] w=0$

$2 d(u) v[w, u]=0$

$d(u) v[w, u]=0$, for all $u, v, w \in U$

And hence $(u) U d(u)=(0)$, for all $u, w \in U$.

Thus for each $u \in U$, by lemma 1 we find that either $d(u)=0$ or $[w, u]=0$.

Now let $A=\{u \in U /[w, u]=0$, for all $w \in U\}$ and $B=\{u \in U / d(u)=0\}$.

Then $A$ and $B$ are additive subgroups of $U$ and $U=A \cup B$. But a group cannot be a union of two its proper subgroups, and hence $U=A$ or $U=B$. If $U=A$, then $d(u)=0$.

Thus by lemma 2, we get the required result. On the other hand if $[w, u]=0$ for all $u, w \in U$, then by lemma 6 , we get $U \subseteq Z(R)$. This completes the proof of the theorem.

Using the same techniques with necessary variations, we can prove the following corollary even without the characteristic assumption on the ring.

\section{Corollary 1}

Let $R$ be a prime ring. If $R$ admits a nonzero left multiplicative generalized derivation $F$ with $d$ such that $[F(x), x]=0$, for all $x \in R$, and if $d \neq 0$, then $R$ is a commutative.

\section{Theorem 2}

Let $R$ be a 2-torsion free prime ring and $U$ a nonzero lie ideal of $R$ such that $u^{2} \in U$, for all $u \in U$. If $R$ admits a left multiplicative generalized derivation $F$ with $d$ such that $F([u, v])=[u, v]$, for all $u, v \in U$, and if $F=0$ or $d \neq 0$, then $U \subseteq Z(R)$.

\section{Proof}

Given that $F$ is a left multiplicative generalized derivation of $R$ such that,

$F([u, v])=[u, v]$, for all $u, v \in U$

If $F=0$, then $[u, v]=0$, for all $u, v \in U$.

Thus lemma 6, we get the required result. 
Now, onward we assume that $F \neq 0$. Suppose on contrary that $U \nsubseteq Z(R)$.

We have $F([u, v])=[u, v]$, for all $u, v \in U$.

$$
\begin{aligned}
& F(u v-v u)=[u, v] \\
& F(u v)-F(v u)=[u, v] \\
& u F(v)+d(u) v-v F(u)-d(v) u=[u, v]
\end{aligned}
$$

Replacing $v$ by $2 u v$ in equation (4) and using the fact that char $(R) \neq 2$, we find that

$$
\begin{aligned}
& u F(2 u v)+d(u) 2 u v-2 u v F(u)-d(2 u v) u=[u, 2 u v] \\
& 2 u^{2} F(v)+2 u d(u) v+2 d(u) u v-2 u v F(u)-2 d(u) v u-2 u d(v) u=2 u[u, v]+2[u, u] v \\
& 2 u(u F(v)+d(u) v-v F(u)-d(v) u)+2 d(u)[u v-v u]=2 u[u, v]
\end{aligned}
$$

Using equation (4) then, we get

$d(u)[u, v]=0$, for all $u, v \in U$.

Again replacing $v$ by $2 v w$ then, we get

$d(u)[u, 2 v w]=0$, for all $u, v, w \in U$.

$d(u) 2 v[u, w]+d(u) 2[u, v] w=0$

$2 d(u) v[u, w]+2 d(u)[u, v] w=0$

$d(u) v[u, w]=0$, for all $u, v, w \in U$.

And hence $d(u) \cup[u, w]=0$, for all $u, w \in U$.

Thus for each $u \in U$, by lemma 1 , either $d(u)=0$ or $[u, w]=0$.

Now, let $U_{1}=\{u \in U / d(u)=0\}$ and $U_{2}=\{u \in U /[u, w]=0$, for all $w \in U\}$.

Then $U_{1}$ and $U_{2}$ both are additive subgroups of $U$ and $U_{1} \cup U_{2}=U$. Thus either $U_{1}=U$ or $U_{2}=U$. If $U_{2}=U$, then $[U, W]=0$, for all $u, w \in U$. Hence by lemma 6 , we get $U \subseteq Z(R)$, contradiction. On the other hand if $U_{1}=U$, then $d(U)=0$, for all $u \in U$. Thus by lemma 2 , we get $U \subseteq Z(R)$, again a contradiction. This completes the proof of the theorem.

\section{Theorem 3}

Let $R$ be a 2-torsion free prime ring and $U$ a nonzero lie ideal of $R$ such that $u^{2} \in U$, for all $u \in U$. If $R$ admits a left multiplicative generalized derivation $F$ with $d$ such that $F([u, v])+[u, v]=0$, for all $u, v \in U$, and if $F=0$ or $d \neq$ 0 , then $U \subseteq Z(R)$.

\section{Proof}

Given that $F$ is a left multiplicative generalized derivation of $R$ such that,

$F([u, v])+[u, v]=0$, for all $u, v \in U$ 
If $F=0$, then $[u, v]=0$, for all $u, v \in U$.

Thus lemma 6, we get the required result.

Now, onward we assume that $F \neq 0$. Suppose on contrary that $U \nsubseteq Z(R)$.

We have $F([u, v])+[u, v]=0$, for all $u, v \in U$.

$$
\begin{aligned}
& F(u v-v u)+[u, v]=0 \\
& F(u v)-F(v u)+[u, v]=0 \\
& u F(v)+d(u) v-v F(u)-d(v) u+[u, v]=0
\end{aligned}
$$

Replacing $v$ by $2 u v$ in equation (6) and using the fact that char $(R) \neq 2$, we find that

$$
\begin{aligned}
& u F(2 u v)+d(u) 2 u v-2 u v F(u)-d(2 u v) u+[u, 2 u v]=0 \\
& 2 u^{2} F(v)+2 u d(u) v+2 d(u) u v-2 u v F(u)-2 d(u) v u-2 u d(v) u+2 u[u, v]+2[u, u] v=0 \\
& 2 u(u F(v)+d(u) v-v F(u)-d(v) u+[u, v])+2 d(u)[u v-v u]=0
\end{aligned}
$$

Using equation (6) then, we get

$d(u)[u, v]=0$, for all $u, v \in U$.

The equation (7) is same as equation (5) in theorem 2. Thus, by same argument of theorem 2 , we can conclude the result is there. Thus the proof is completed.

\section{Theorem 4}

Let $R$ be a 2-torsion free prime ring and $U$ a nonzero lie ideal of $R$ such that $u^{2} \in U$, for all $u \in U$. If $R$ admits a left multiplicative generalized derivation $F$ with $d$ such that $F(u o v)=u o v$, for all $u, v \in U$, and if $F=0$ or $d \neq 0$, then $U \subseteq Z(R)$

\section{Proof}

Given that $F$ is a left multiplicative generalized derivation of $R$ such that,

$F(u o v)=u o v$, for all $u, v \in U$

If $F=0$, then we have

$u o v=0$, for all $u, v \in U$

Replacing $v$ by $2 w v$ in equation (8) and using equation (8) then, we get

$u o(2 w v)=0$

$2 w(u o v)+2[u, w] v=0$

$2[u, w] v=0$

$[u, w] v=0$, for all $u, v, w \in U$

Again replace $v$ by $[u, r]$, we get 
$[u, w][u, r]=0$, for all $u, w \in U, r \in R$.

For any $s \in R$, we replacing $r$ by $r s$ then, we get

$[u, w][u, r s]=0$

$[u, w] r[u, s]+[u, w][u, r] s=0$

$[u, w] R[u, s]=0$, for all $u, w \in U, r, s \in R$.

Thus, in particular we have $[u, w] R[u, w]=0$, for all $u, w \in U, r \in R$.

Thus, primness of $R$ yields that $[u, w]=0$, for all $u, w \in U$.

And hence by lemma 6, we get the required result.

Therefore now onward we shall assume that $F \neq 0$. Suppose on contrary that $U \nsubseteq Z(R)$.

We have, $F(u o v)=u o v$, for all $u, v \in U$.

$F(u v+v u)=u o v$

$F(u v)+F(v u)=u o v$

$u F(v)+d(u) v+v F(u)+d(v) u=u o v$, for all $u, v \in U$.

We replacing $v$ by $2 u v$ in equation (9) then, we get

$$
\begin{aligned}
& u F(2 u v)+d(u) 2 u v+2 u v F(u)+d(2 u v) u=u o 2 u v . \\
& 2 u^{2} F(v)+2 u d(u) v+2 d(u) u v+2 u v F(u)+2 d(u) v u+2 u d(v) u=2 u(u o v)+2[u, u] v \\
& 2 u(u F(v)+d(u) v+v F(u)+d(v) u-(u o v))+2 d(u) u v+2 d(u) v u=0
\end{aligned}
$$

Using equation (9), then we get

$$
\begin{aligned}
& 2 d(u) u v+2 d(u) v u=0 \\
& 2 d(u)(u v+v u)=0 \\
& d(u)(u o v)=0, \text { for all } u, v \in U .
\end{aligned}
$$

Again replace $v$ by $2 v w$, we get

$$
\begin{aligned}
& d(u)(u o 2 v w)=0 \\
& d(u)(2(u \circ v) w-2 v[u, w])=0 \\
& 2 d(u)(u \circ v) w-2 d(u) v[u, w]=0
\end{aligned}
$$

Using equation (10), then we get

$-2 d(u) v[u, w]=0$

$d(u) v[u, w]=0$, for all $u, v, w \in U$.

Note that the equation (11) as the same arguments given in the proof of theorem 3, hence repeating the same 
process we get the required result. Thus the proof is completed.

\section{Theorem 5}

Let $R$ be a 2-torsion free prime ring and $U$ a nonzero lie ideal of $R$ such that $u^{2} \in U$, for all $u \in U$. If $R$ admits a left multiplicative generalized derivation $F$ with $d$ such that $F(u o v)+u o v=0$, for all $u, v \in U$, and if $F=0$ or $d \neq 0$, then $U \subseteq Z(R)$.

\section{Proof}

Given that $F$ is a left multiplicative generalized derivation of $R$ such that,

$F(u o v)+u o v=0$, for all $u, v \in U$

If $F=0$, then we have

$u o v=0$, for all $u, v \in U$

Replacing $v$ by $2 w v$ in equation (12) and using equation (12) then, we get

$u o(2 w v)=0$

$2 w(u o v)+2[u, w] v=0$

$2[u, w] v=0$

$[u, w] v=0$, for all $u, v, w \in U$.

Again replace $v$ by $[u, r]$, we get

$[u, w][u, r]=0$, for all $u, w \in U, r \in R$.

For any $s \in R$, we replacing $r$ by $r s$ then, we get

$[u, w][u, r s]=0$

$[u, w] r[u, s]+[u, w][u, r] s=0$

$[u, w] R[u, s]=0$, for all $u, w \in U, r, s \in R$.

Thus, in particular we have $[u, w] R[u, w]=0$, for all $u, w \in U, r \in R$.

Thus, primness of $R$ yields that $[u, w]=0$, for all $u, w \in U$.

And hence by lemma 6, we get the required result.

Therefore now onward we shall assume that $F \neq 0$. Suppose on contrary that $U \nsubseteq Z(R)$.

We have, $F(u o v)+u o v=0$, for all $u, v \in U$.

$F(u v+v u)+u o v=0$

$F(u v)+F(v u)+u o v=0$

$u F(v)+d(u) v+v F(u)+d(v) u+u o v=0$, for all $u, v \in U$.

We replacing $v$ by $2 u v$ in equation (13) then, we get 


$$
\begin{aligned}
& u F(2 u v)+d(u) 2 u v+2 u v F(u)+d(2 u v) u+u o(2 u v)=0 . \\
& 2 u^{2} F(v)+2 u d(u) v+2 d(u) u v+2 u v F(u)+2 d(u) v u+2 u d(v) u+2 u(u o v)+2[u, u] v=0 \\
& 2 u(u F(v)+d(u) v+v F(u)+d(v) u+(u o v))+2 d(u) u v+2 d(u) v u=0
\end{aligned}
$$

Using equation (13), then we get

$$
\begin{aligned}
& 2 d(u) u v+2 d(u) v u=0 \\
& 2 d(u)(u v+v u)=0 \\
& d(u)(u o v)=0, \text { for all } u, v \in U .
\end{aligned}
$$

The equation (14) is same as equation (10) in theorem 4. Thus, by same argument of theorem 4, we can conclude the result is there. Thus the proof is completed.

\section{IDEALS AND LEFT MULTIPLICATIVE GENERALIZED DERIVATION ON PRIME RINGS}

\section{Theorem 6}

Let $R$ be a prime ring and $I$ a nonzero ideal of $R$. If $R$ admits a left multiplicative generalized derivation $F$ with $d$ such that $F(x \circ y)=x o y$ holds for all $x, y \in I$, and if $F=0$ or $d \neq 0$, then $R$ is a commutative.

\section{Proof}

Given that $F$ is a left multiplicative generalized derivation of $R$ such that,

We have, $F(x o y)=x o y$, for all $x, y \in I$.

If $F=0$, then $x o y=0$, for all $x, y \in I$.

Replacing $y$ by $z y$ in above equation then, we get

$x o(z y)=0$

$z(x o y)+[x, z] y=0$

$[x, z] y=0$, for all $x, y, z \in I$.

And hence $[x, z] R I=(0)$, for all $x, z \in I$.

Since $I \neq(0)$, and $R$ is a prime, we get

$[x, z]=0$, for all $x, z \in I$.

And hence by lemma 5, $R$ is a commutative. Hence onward we assume that $F \neq 0$.

We have, $F(x o y)=x o y$, for all $x, y \in I$.

$$
\begin{aligned}
& F(x y+y x)=x o y \\
& F(x y)+F(y x)=x o y \\
& x F(y)+d(x) y+y F(x)+d(y) x=x o y, \text { for all } x, y \in I .
\end{aligned}
$$

Replacing $y$ by $x y$ in equation (15) then, we get 
$x F(x y)+d(x) x y+x y F(x)+d(x y) x=x o x y$

$x^{2} F(y)+x d(x) y+d(x) x y+x y F(x)+d(x) y x+x d(y) x=x(x o y)+[x, x] y$

$x(x F(y)+d(x) y+y F(x)+d(y) x-(x \circ y))+d(x) x y+d(x) y x=0$

Using equation (15), we get

$d(x) x y+d(x) y x=0$

$d(x)(x y+y x)=0$

$d(x)(x \circ y)=0$, for all $x, y \in I$.

Again replace $y$ by $y z$ in above equation then, we get

$$
\begin{aligned}
& d(x)(x \circ y z)=0 \\
& d(x)((x \circ y) z-y[x, z])=0 \\
& d(x)(x \circ y) z-d(x) y[x, z]=0
\end{aligned}
$$

Using equation (16) then, we get

$d(x) y[x, z]=0$, for all $x, y, z \in I$.

And hence $d(x) R I[x, z]=0$, for all $x, z \in I$.

Thus primness of $R$ forces that for each $x \in I$ either $d(x)=0$ or $I[x, z]=0$, for all $x, z \in I$. The set of $x \in I$ for which there two properties hold are additive sub groups of $I$ whose union is $I$, and therefore, $d(x)=0$, for all $x \in I$ or $I[x, z]=0$, for all $x, z \in I$. If $I[x, z]=0$, for all $x, z \in I$, then $I R[x, z]=0$. Since $I \neq 0$, we find that $[x, z]=0$, for all $x, z \in I$, and hence by lemma $5, R$ is a commutative. On the other hand if $d(x)=0$, for all $x \in I$, then implies that $[d(x), x]=0$, for all $x \in I$, and hence by lemma $4, R$ is a commutative.

\section{Theorem 7}

Let $R$ be a prime ring and $I$ a nonzero ideal of $R$. If $R$ admits a left multiplicative generalized derivation $F$ with $d$ such that $F(x o y)+x o y=0$ holds for all $x, y \in I$, and if $F=0$ or $d \neq 0$, then $R$ is a commutative.

\section{Proof}

Given that $F$ is a left multiplicative generalized derivation of $R$ such that,

We have, $F(x o y)+x o y=0$, for all $x, y \in I$.

If $F=0$, then $x o y=0$, for all $x, y \in I$.

Replacing $y$ by $z y$ in above equation then, we get

$x o(z y)=0$

$z(x \circ y)+[x, z] y=0$

$[x, z] y=0$, for all $x, y, z \in I$. 
And hence $[x, z] R I=(0)$, for all $x, z \in I$.

Since $I \neq(0)$, and $R$ is a prime, we get

$[x, z]=0$, for all $x, z \in I$.

And hence by lemma 5, $R$ is a commutative. Hence onward we assume that $F \neq 0$.

We have, $F(x o y)+x o y=0$, for all $x, y \in I$.

$F(x y+y x)+x o y=0$

$F(x y)+F(y x)+x o y=0$

$x F(y)+d(x) y+y F(x)+d(y) x+x o y=0$, for all $x, y \in I$.

Replacing $y$ by $x y$ in equation (18) then, we get

$$
\begin{aligned}
& x F(x y)+d(x) x y+x y F(x)+d(x y) x+x o x y=0 \\
& x^{2} F(y)+x d(x) y+d(x) x y+x y F(x)+d(x) y x+x d(y) x+x(x o y)+[x, x] y=0 \\
& x(x F(y)+d(x) y+y F(x)+d(y) x+(x o y))+d(x) x y+d(x) y x=0
\end{aligned}
$$

Using equation (18), we get

$$
\begin{aligned}
& d(x) x y+d(x) y x=0 \\
& d(x)(x y+y x)=0 \\
& d(x)(x \circ y)=0, \text { for all } x, y \in I .
\end{aligned}
$$

The equation (19) is same as equation (16) in theorem 6. Thus, by same argument of theorem 6, we can conclude the result is there. Thus the proof is completed.

\section{CONCLUSIONS}

- In this article we proved some theorems (1)-(5) that are interms of a nonzero left multiplicative generalized derivation satisfies certain condition implies .

- In the next section we proved two theorems about commutativity see Theorem (6)-(7).

\section{REFERENCES}

1. Ashraf, M., \& Rehman, N.(2018)On commtativity of rings with derivations, Results Math.(to appear).

2. Awtar, R. (1984). Lie structure in prime rings with derivations, Publ. Math, Debrecen, 31, 209-215.

3. Bell, H. E., \& Martindale, W. S. (1987). Centralizing mapping of semiprime rings, Canad. Math. Bull, 30,92-101.

4. Bergen, J., Herstein I. N., \&Kerr. J. W. (1981). Lie ideals and derivations of prime rings, J. Algebra,71, 259-267.

5. Bresar, M.(1993). Commuting traces of biadditive mappings, commutativity-preserving mappings and Lie mappings, Trans. Math. Soc, 335, 525-546.

6. Bresar, M. (1993). Centralizing mappings and derivations in prime rings, Journal of Algebra, 156, 385-394.

7. Daif, M. N., \& Bell, H. E. (1992). Remarks on derivations on semiprime rings, Internat. J. Math. Math. Sci., 15, 1, $205-206$. 
8. Herstein. I. N. (1969). Topics in ring theory, Univ. of Chicago Press, Chicago.

9. Herstein. I. N. (1976). Rings with involution, University Chicago press, Chicago.

10. Hvala, B. (1998). Generalized derivations in prime rings, Comm. Algebra, 26, 4, 1147-1166.

11. Mayne, J. H. (1984). Centralizing mappings of prime rings, Canad. Math. Bull., 27, 1, 122-126.

12. Nadeem-ur-Rehman.(2002). On commutativity of rings with generalized derivations, Math. J. Okayama Univ., 44, 43-49.

13. Posner E. C. (1957). Derivations in prime rings, Proc. Amer. math. Soc. 8, 1093-1100.

14. Subba Reddy C. J., Vijay Kumar, V., \& Mallikarjuna Rao, S.(2015). Left multiplicative generalized derivations on right ideal in semiprime rings, IOSR Journal of Mathematics., 11, 60-63.

15. Reddy, C. J. S., \& Hemavathi, K. Right Reverse Derivations on Prime Rings.

16. Subba Reddy C. J., Vijay Kumar, V., Mallikarjuna Rao, S. (2015). Left multiplicative generalized derivations in

17. semiprime rings, International Journal of Scientific Innovative Mathematical Research., 3,575-579.

18. Subba Reddy C. J., Mallikarjuna Rao S., \& Mahesh Kumar, T.(2014). Left multiplicative generalized derivations on semiprime rings, Mathematical Sciences International Research Journal., 3, 859-861.

19. Subba Reddy C. J., \& Hemavathi, K. (2014). Left multiplicative generalized derivationon lie ideals in prime rings, International Journal of Mathematics Comutation. 2, 12-18. 